\title{
Running Control of a Planar Biped Robot based on Energy-Preserving Strategy
}

\author{
Sang-Ho Hyon \\ Department of Bioengineering and Robotics \\ Graduate School of Engineering, Tohoku University \\ Aramaki-Aza-Aoba 01, Sendai 980-8579, Japan \\ Email: sangho@ieee.org
}

\author{
Takashi Emura \\ Department of Bioengineering and Robotics \\ Graduate School of Engineering, Tohoku University \\ Aramaki-Aza-Aoba 01, Sendai 980-8579, Japan \\ Email: emura@emura.mech.tohoku.ac.jp
}

\begin{abstract}
In this paper, we extend running controller of passive one-legged hopper to a planar biped robot with torso, and evaluate the controller on simulations. The controller is derived based on energy-preserving strategy and it actually preserves mechanical energy at touchdown. Interestingly, zero dynamics of decoupling controller (dynamics about pair of controlled leg) is found to be stable. Combining simple attitude controller at stance phase generates stable periodic running gaits of arbitral period. The control performance is shown to be better than a simple PD-feedback control of leg placement.
\end{abstract}

\section{INTRODUCTION}

\section{A. Background}

After the Raibert's excellent works [1], running robots have been widely studied both experimentally [2][3][4][5][6] and theoretically [7][8]. On the other hand, recently there are many studies on biped humanoid robots with the aim of practical application. Therefore, enhancing the mobility of them is important target to be reached. Running control of biped humanoid robots is included in such targets. Energy-efficient control of fast running is especially crucial for autonomous humanoid robots because it directly extends operation time.

In this connection, there are some remarkable researches on energy-efficient running control. Tompson and Raibert showed that spring-driven one-legged hopping robot can hop without any inputs, provided if the initial conditions were appropriately chosen [9]. Ahmadi and Buehler applied Raibert's algorithm to this robot and realized energy-efficient hopping in simulation and experiment [10]. François and Samson derived a rather systematic controller based on the general control method used in nonlinear oscillatory system [11].

\section{B. Energy-preserving controller for one-legged hopper}

Motivated from their works, we proposed, in [12], alternative controller based on its energy analysis for a planar onelegged robot shown in Fig. 1.

The underlining principle is energy-preserving control strategy. This means the controller preserves system energy as much as possible. The most important reason why we use this strategy is; if the system energy is preserved, it is expected that the system autonomously generates natural periodic gaits, just as some class of Hamiltonian system exhibit natural periodic orbit [13]. Instead of depending on some pre-calculated

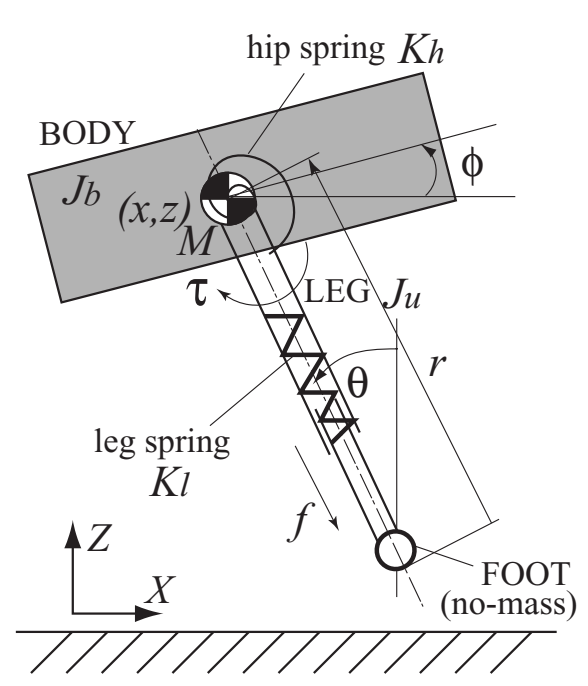

Fig. 1. Passive one-legged hopper

periodic solutions, or target (desired) dynamics, analysis on energy change of the original nonlinear hybrid system were utilized as shown below.

First, we choose desired touchdown angle $\theta_{d}$ and angular velocity $\dot{\theta}_{d}$ at the moment of lift-off to meet the following energy non-dissipation condition:

$$
\mu_{t d-}:=\dot{x}_{t d-} \cos \theta_{t d}+\dot{z}_{t d-} \sin \theta_{t d}+r_{0}=\dot{\theta}_{t d-}=0
$$

Subscript "td" means just the moment of touchdown and "td-" or "td+" mean just before / after touchdown hereinafter. Since the energy change between just before and after touchdown is calculated as

$$
E_{t d+}-E_{t d-}=-\frac{M J_{l}}{2\left(J_{l}+M r_{0}^{2}\right)} \mu_{t d-}^{2},
$$

condition (1) means there is no energy exchange between the robot and the ground, provided if no control input applied during stance phase. Having determined $\theta_{d}$ and $\dot{\theta}_{d}$, finally we can apply simple linear dead-beat controller because the flight dynamics is integrable.

As a result, interesting quasi-periodic gaits, which can be seen in some Hamiltonian system, were found, and both period 

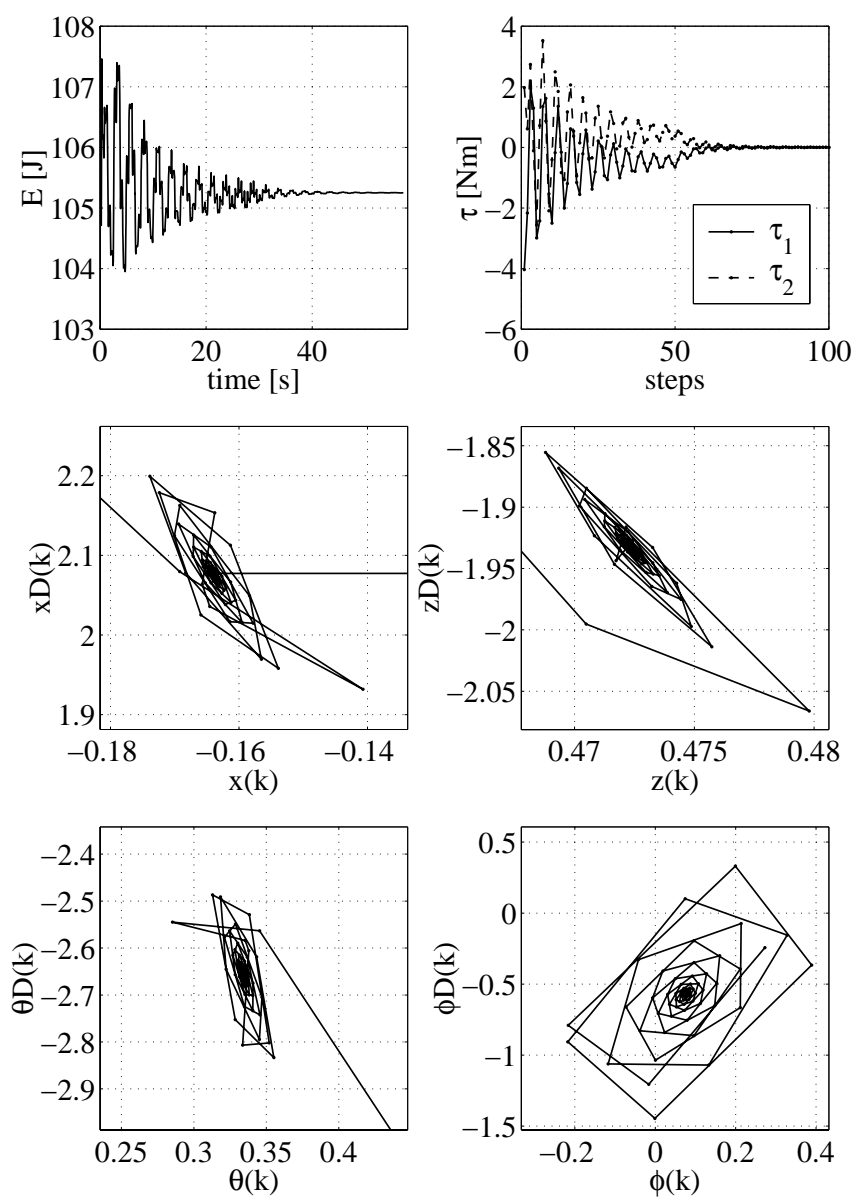

Fig. 2. Simulation results of the orbital stabilization controller and the spring stiffness adaptation. The top two graph shows time evolutions of energy level $(E)$ and control inputs $\left(\tau_{1}\right.$ and $\tau_{2}$. The lower four graphs represent the selected images of the Poincaré map, where notation " $D$ " represents the time derivatives of preceding variables. Please note that the position $x$ is manually reset at each iterative crossing of the section, for visibility. The input minimization via spring stiffness adaptation law is activated from the 60th step. You can see the images of Poincaré Map asymptotically converge to one fixed point and the control inputs eventually converge to zero!

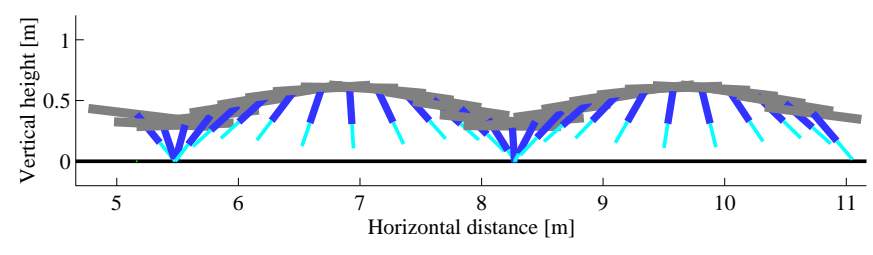

Fig. 3. Subsequent two steps of 1-periodic passive running gait. Running speed is $5[\mathrm{~m} / \mathrm{s}]$. The robot moves from left to the right

stabilization and one-periodic passive running were achieved in simulation. It was also found that an adaptive control of touchdown angle, which is similar to the delayed feedback controller for chaotic system [15], can asymptotically stabilize these quasi-periodic gaits to desired periodic ones. Especially for 1-periodic gait, by using some additional adaptive controllers, the robot eventually hops without any control inputs, that is, complete passive running is obtained. Fig. 2 is an example of simulation results, which shows an adaptation control law achieves complete passive running. Fig. 3 is the stick pictures of high-speed passive running at $5[\mathrm{~m} / \mathrm{s}]$. Complete description and results can be found in the literature [14].

\section{Paper organization}

The purpose of this paper it to extend the controller of passive one-legged hopper to biped robots. This was partially done on a 3D biped model in [17], where a rotor rotating around yaw-axis of torso was introduced. In this paper, we consider a highly nonlinear planar biped model having massive legs and torso and try to achieve stable periodic running gaits of it. Specifically, we derive dead-beat controller at flight phase based on the energy-preserving strategy to preserve mechanical energy at touchdown. Then, we combine some stance-phase controllers to get stable running gaits.

The paper is organized as follows: Section II introduces our new biped running robot and the equations of motion of simplified model are given. Section III extends the controller described in Section I to a planar biped robot with torso, by introducing nonlinear decoupling control and target dynamics. Section IV shows simulation results of biped running. Section $\mathrm{V}$ concludes this paper.

\section{A PLANAR BIPED ROBOT}

Figure 4 shows a CAD model of newly developed planar biped robot, SkipperII. The robot has two springy telescopic legs swinging around hip joints. Leg actuators are mounted parallel to the leg spring. Overall height of the robot is 0.75 [m] and the total weight is about $7[\mathrm{~kg}]$. The hardware design and experiments will be presented elsewhere.

Figure 5 shows the definition of mathematical model. The generalized coordinates are defined as the position of center of gravity (CoG), $x=\left(x_{g}, z_{g}\right)^{T} \in R^{2}$, the attitude of the torso, $\phi \in R^{1}$, and joint angles, $\psi=\left(\psi_{1}, \psi_{2}\right)^{T} \in R^{2} . M_{b}$ and $m$ are the mass of torso and leg respectively. $I$ and $J$ are the moment of inertia about $\mathrm{CoG}$ of the torso and $\mathrm{CoG}$ of the leg respectively. All principal axes of each rigid part are coincident with their center axes. Table I shows the physical parameters, together with the values used in later simulations. This model is highly nonlinear because it has massive legs and torso, whose CoG are located away the hip joint.

Additionally, the following assumptions are imposed on the model:

(A) Mass of the foot (unsprung mass) is negligible

(B) The foot does not bounce back, nor slip the ground (inelastic impulsive impact) 


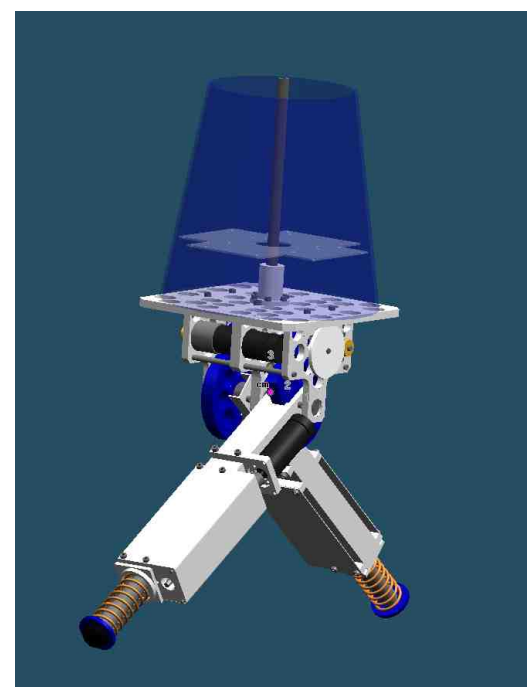

Fig. 4. CAD model of a new planar biped robot, SkipperII

(C) The springs are mass-less and non-dissipating

A running motion is composed of successive phase transitions; stance $\rightarrow$ flight $\rightarrow$ touchdown $\rightarrow$ stance $\rightarrow \cdots$. Below we show the equations of motion at each phase in abbreviated from.

\section{A. Equations of motion at stance / flight phase}

The equations of motion at stance phase (right leg support) can be derived by Lagrangian formulation.

$$
\begin{aligned}
& {\left[\begin{array}{cc|cc}
M & 0 & \multicolumn{2}{|c}{0_{2 \times 3}} \\
0 & M & N_{11} & N_{12} \\
\hline 0_{3 \times 2} & N_{21} & M_{22}
\end{array}\right]\left[\begin{array}{c}
\ddot{x} \\
\hline \ddot{\phi} \\
\ddot{\psi}
\end{array}\right]} \\
& =\left[\begin{array}{c}
0 \\
-M g \\
\hline H_{1} \\
H_{2}
\end{array}\right]+\left[\frac{\lambda}{0_{3 \times 1}}\right]+\left[\begin{array}{c}
f \\
0 \\
\tau
\end{array}\right]
\end{aligned}
$$

Here, $M=2 m+M_{b}$ is the total mass and $g$ is gravity acceleration. $N_{11} \in R^{1}, N_{21}=N_{12}^{T} \in R^{1 \times 2}$, and $N_{22} \in$ $R^{2 \times 2}$ are inertia matrix. $H_{1} \in R^{1}$ and $H_{2} \in R^{2 \times 1}$ are nonlinear terms including centrifugal force, Coriolis force, gravity force and the leg spring forces.

The generalized foces are $f=\left(f_{1}, f_{2}\right)^{T} \in R^{2}$, where $f_{1}$ and $f_{2}$ are the forces of leg actuators, and $\tau=\left(\tau_{1}, \tau_{2}\right)^{T} \in R^{2}$, where $\tau_{1}$ and $\tau_{2}$ are the torques of hip actuators, and $\lambda=$ $\left(\lambda_{x}, \lambda_{z}\right)^{T} \in R^{2}$ represents ground reaction forces. Thanks

TABLE I

ROBOT PARAMETERS

\begin{tabular}{|c|c|c|}
\hline Variables & Unit & Values \\
\hline$L, L_{b}, r_{0}$ & $\mathrm{~m}$ & $0.16,0.2,0.08$ \\
$M_{b}, m$ & $\mathrm{~kg}$ & $4.11,2.13$ \\
$I, J$ & $\mathrm{kgm}^{2}$ & $0.05,0.015$ \\
$K_{l}$ & $\mathrm{~N} / \mathrm{m}$ & 3000 \\
\hline
\end{tabular}

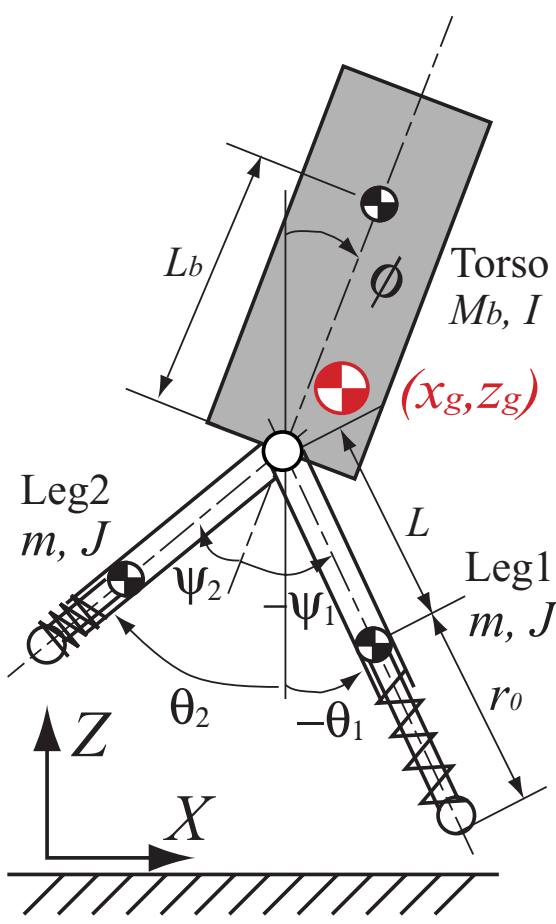

Lateral view

Fig. 5. Definition of mathematical model

to assumption (C), we assume $f=0$ throughout this paper. Dynamics of flight phase can be obtained by removing $\lambda$ and $f$ from (3).

\section{B. Equations of motion at touchdown phase}

From assumption (B), touchdown equation can be expressed as Lagrange's impulsive equation.

$$
\left[\begin{array}{cc|cc}
M & 0 & \multicolumn{2}{|c}{0_{2 \times 3}} \\
0 & M & \\
\hline 0_{3 \times 2} & N_{11} & N_{12} \\
N_{21} & M_{22}
\end{array}\right]\left[\begin{array}{c}
\Delta \dot{x} \\
\hline \Delta \dot{\phi} \\
\Delta \dot{\psi}
\end{array}\right]=E(q)^{T} \hat{\lambda}_{p},
$$

where $\Delta \dot{x}:=\dot{x}_{t d+}-\dot{x}_{t d-}$ means instantaneous velocity changes, so does $\Delta \dot{e}$ and $\Delta \dot{p}$. $\hat{\lambda}_{p} \in R^{1}$ is the constraint impulse perpendicular to leg axis, associated with instantaneous velocity constraint at touchdown:

$$
\left[\begin{array}{lllll}
\cos \theta_{1} & \sin \theta_{1} & 0 & -\left(L+r_{0}\right) & 0
\end{array}\right]\left[\begin{array}{c}
\dot{x}_{0} \\
\dot{\phi} \\
\dot{\psi}
\end{array}\right]=0
$$

where $x_{0} \in R^{2}$ is the hip position, and $r_{0}$ is the nominal leg length. Note that the constraint impulse along leg axis is completely absorbed by the leg spring, due to the assumption (A) and (C), and hence does not appears in (4). After coordinate transformation from $x_{0}$ to $x$, (5) becomes to

$$
E(q)\left[\begin{array}{c}
\dot{x} \\
\dot{\phi} \\
\dot{\psi}
\end{array}\right]=0
$$


where $E(q) \in R^{1 \times 5}$ is jacobian of velocity constraint, which appears in (4).

\section{CONTROLLER IMPLEMENTATION VIA DECOUPLING}

\section{A. Stance phase controller}

It is well known that a torso mounted below the hip joint has passive stability [3]. In most of biped robots, however, the torso is located above the hip joint like Fig. 5. In contrast to one-legged robot, in which no control inputs are applied, the biped robot cannot hold its torso upright posture without pitch control, because of reaction forces from hip joints.

Here we are temporarily using simple pitch control, regardless of expense of actuator energy:

$$
\tau_{1}=-K_{1 p} \phi-K_{1 d} \dot{\phi}
$$

where $K_{1 p} \geq 0$ and $K_{1 d} \geq 0$. The supporting leg is assumed to be Leg1 indicated in Fig. 5 .

For swinging leg (Leg2), we are also temporarily controlling it by

$$
\tau_{2}=-K_{2 p}\left(\psi_{2}+\psi_{1}\right)-K_{2 d}\left(\dot{\psi}_{2}+\dot{\psi}_{1}\right)
$$

Under this controller, counter oscillation of each leg is expected during running.

Remarks: $\quad$ Since we are not satisfied with this somewhat "factitious" controller that can deteriorate some useful intrinsic nonlinear dynamics at stance phase. Specifically, as long as we use that controller, internal energy cannot be preserved. The solution will be introducing hip springs that can preserve energy and produce oscillatory motion, as in the case of passive one-legged model.

\section{B. Flight phase controller}

To apply the controller of one-legged model to the biped, decoupling control and target dynamics are introduced. Here we suppose the next touchdown leg is Leg1.

First, the equation of motion (3) is decoupled using a new control input. Rewriting the lower part of (3), we obtains

$$
\begin{gathered}
N_{11} \ddot{\phi}+N_{12} \ddot{\psi}+H_{1}=0 \\
N_{21} \ddot{\phi}+N_{22} \ddot{\psi}+H_{2}=\tau .
\end{gathered}
$$

Equation (9) represents time differential of conservation law of angular velocity around $\mathrm{CoG}$ of the robot. Rearranging these equations, we obtain

$$
\left[\begin{array}{l}
\ddot{\phi} \\
\hline \dot{\psi}
\end{array}\right]=\left[\begin{array}{ll}
N_{11} & N_{12} \\
N_{21} & N_{22}
\end{array}\right]^{-1}\left[\frac{-H_{1}}{-H_{2}+\tau}\right] .
$$

Then we decouple the lower part of this equation by new control inputs $u_{1}$ and $u_{2}$ defined later (partial feedback lenearization):

$$
\left[\begin{array}{c}
\ddot{\psi}_{1} \\
\ddot{\psi_{2}}
\end{array}\right]=\left[\begin{array}{l}
u_{1} \\
u_{2}
\end{array}\right]
$$

This equation means we can control $\ddot{\psi}$ arbitrarily by new control inputs $u_{1}$ and $u_{2}$.
Next, we define a target dynamics. Conservation law of the angular momentum around $\mathrm{CoG}$ can be expressed as

$$
J_{0} \dot{\phi}+J_{1} \dot{\psi}_{1}+J_{2} \dot{\psi}_{2}=P_{0}
$$

where $J_{i}(i=0,1,2)$ is nonlinear inertia terms and $P_{0}$ means initial angular momentum of flight phase. If we chose

$$
\dot{\psi}_{2}=\frac{1}{J_{2}}\left(P_{0}-J_{0} \dot{\phi}_{0}-J_{1} \dot{\psi}_{1}\right)
$$

( $\dot{\phi}_{0}$ is the initial value of $\dot{\phi}$ at flight phase), we get the following target dynamics.

$$
\dot{\phi}=\dot{\phi}_{0}
$$

This means "another first integral of motion is created by a feedback control". Note that it suits normal biped running gait because this constraint holds approximately, when the both legs are swung symmetrically $\left(\dot{\psi}_{1}+\dot{\psi}_{2}=0\right)$. The control input $u_{2}$ that achieve (14) can be easily calculated by the time derivative of (14)

$u_{2}=\frac{d}{d t}\left(\frac{1}{J_{2}}\right) P_{0}-\frac{d}{d t}\left(\frac{J_{0}}{J_{2}}\right) \dot{\phi}_{0}-\frac{d}{d t}\left(\frac{J_{1}}{J_{2}}\right) \dot{\psi}_{1}-\frac{J_{1}}{J_{2}} u_{1}$

Then, the remained task is to determine control input, $u_{1}$ in (16). The control objective is to dead-beat the absolute angle of the swing leg $\theta_{1}$ (Fig. 5) and its velocity $\dot{\theta_{1}}$ to some desired values at given fixed time $T_{v}$ (flight time). As we explained in Section I, desired values are chosen to preserve energy at touchdown. For the biped robot case, condition of energy preservation is given by:

$$
\hat{\lambda}_{p}=0
$$

where $\hat{\lambda}_{p}$ can be calculated explicitly from (4) and (6). For a given flight time $T_{v}$, however, there are many pairs of $\theta_{d}$ and $\dot{\theta}_{d}$, as in the case of one-legged model. For example, to achieve orbital stabilization, $\theta_{d}$ can be chosen as follows.

$$
\theta_{d}(k)=\left\{\begin{array}{lc}
-\frac{1}{2}\left(\theta_{l o}(k)+\theta_{l o}(k-p)\right), & \text { if } k>p \\
-\theta_{l o}(k), & \text { else },
\end{array}\right.
$$

where $k>1$ is the iteration step and $p>1$ is a desired period. This is the same adaptive control law for passive one-legged hopper in [12]. Note that the desired angular velocity $\dot{\theta}_{d}(k)$ is automatically determined by (17) accordingly.

Since (12) is a trivial second order linear ODE, we can easily dead-beat $\psi_{1}$ and $\dot{\psi}_{1}$, by only once-switching of the constant inputs. Note that $\theta_{1}=\psi_{1}+\phi$ and $\dot{\theta}_{1}=\dot{\psi_{1}}+\dot{\phi}$. If we define new variables

$$
\Phi:=\left[\begin{array}{c}
\phi \\
\dot{\phi}
\end{array}\right], \quad \Theta:=\left[\begin{array}{c}
\theta_{1} \\
\dot{\theta_{1}}
\end{array}\right]
$$

and descretize the lower part of (12) using the piecewise constant inputs

$$
u_{1}= \begin{cases}u_{1 a}, & \text { if } 0 \leq t<T_{v} / 2 \\ u_{1 b}, & \text { if } T_{v} / 2 \leq t<T_{v},\end{cases}
$$

the dead-beat control inputs can be calculated as follows: 


$$
\left[\begin{array}{l}
u_{1 a} \\
u_{1 b}
\end{array}\right]=B\left(T_{v}\right)^{-1}\left\{\Psi\left(T_{v}\right)-A\left(T_{v}\right) \Psi(0)\right\}
$$

where

$$
\begin{aligned}
A\left(T_{v}\right) & =\left[\begin{array}{cc}
1 & T_{v} \\
0 & 1
\end{array}\right] \\
B\left(T_{v}\right) & =\left[\begin{array}{cc}
\frac{3}{8} T_{v}^{2} & \frac{1}{8} T_{v}^{2} \\
\frac{1}{2} T_{v} & \frac{1}{2} T_{v}
\end{array}\right] \\
\Psi\left(T_{v}\right) & =\Theta_{d}-A\left(T_{v}\right) \Phi(0)
\end{aligned}
$$

and $\Theta_{d}$ is the desired value of $\Theta$.

\section{Simulation Results}

\section{A. Stable periodic running}

We have simulated the action of the controller proposed in the previous section. The simulation starts from stance phase of Leg1 and initial height $z_{g 0}$, or initial vertical velocity $\dot{z}_{g 0}$ was chosen large so that the swinging leg does not stub against ground. Fig. 6 shows time evolutions of each state variable and Fig. 7 represents phase portraits. Fig. 8 shows corresponding animation. They show stable 1-periodic running gaits. Control parameters are determined as $K_{p 1}=200, K_{d 1}=50, K_{p 2}=$ $100, K_{d 2}=10$ in (7). Interestingly, dynamics about Leg2 (zero dynamics of decoupling controller) is found to be stable. Actually, the motion of Leg2 indicates counter oscillation of Leg1. Although the running gait seems to have symmetricity, the motion of torso is slightly asymmetric, as recognized from the right top graph of Fig. 7. This asymmetricity become more significant if the feedback gains of (7) become smaller. Without attitude control, the robot falls down after a few steps. The bottom two graphs of Fig. 6 indicate that the attitude control at stance phase requires more power than a dead-beat control at flight phase.

We also simulated stabilization to (unknown) multi-periodic gaits. For example, if we set $p=2$ in (18), we obtain a stable 2-periodic running gait, as shown in Fig. 9.

The controller is also applied to a more precise model using $D A D S$, commercial simulation software (www.cybernet.co.jp), and we obtained almost same results. Animation video will be included in the conference proceedings.

\section{B. Comparison with a simple PD-feedback leg placement}

It is worth comparing the proposed controller with a simple PD-feedback leg placement at flight phase:

$$
\tau=\left\{\begin{array}{l}
-K_{s p 1}\left(\theta_{1}-\theta_{d}\right)-K_{s d 1}\left(\dot{\theta}_{1}\right) \\
-K_{s p 2}\left(\psi_{2}+\psi_{1}\right)-K_{s d 2}\left(\dot{\psi}_{2}\right)
\end{array}\right.
$$

The main difference between this and the proposed controller is whether the angular velocity at touchdown is controlled or not. Fig. 10 shows the phase portraits of the simulation, where control parameter was chosen as $K_{s p 1}=100, K_{s d 1}=$ $10, K_{s p 2}=100, K_{s d 2}=10$. Comparing to Fig. 7 , we can see the symmetricity is damaged large. Although the gaits are still stable owing to (18), energy dissipation always occurs at touchdown, and it makes control inputs quite large at stance
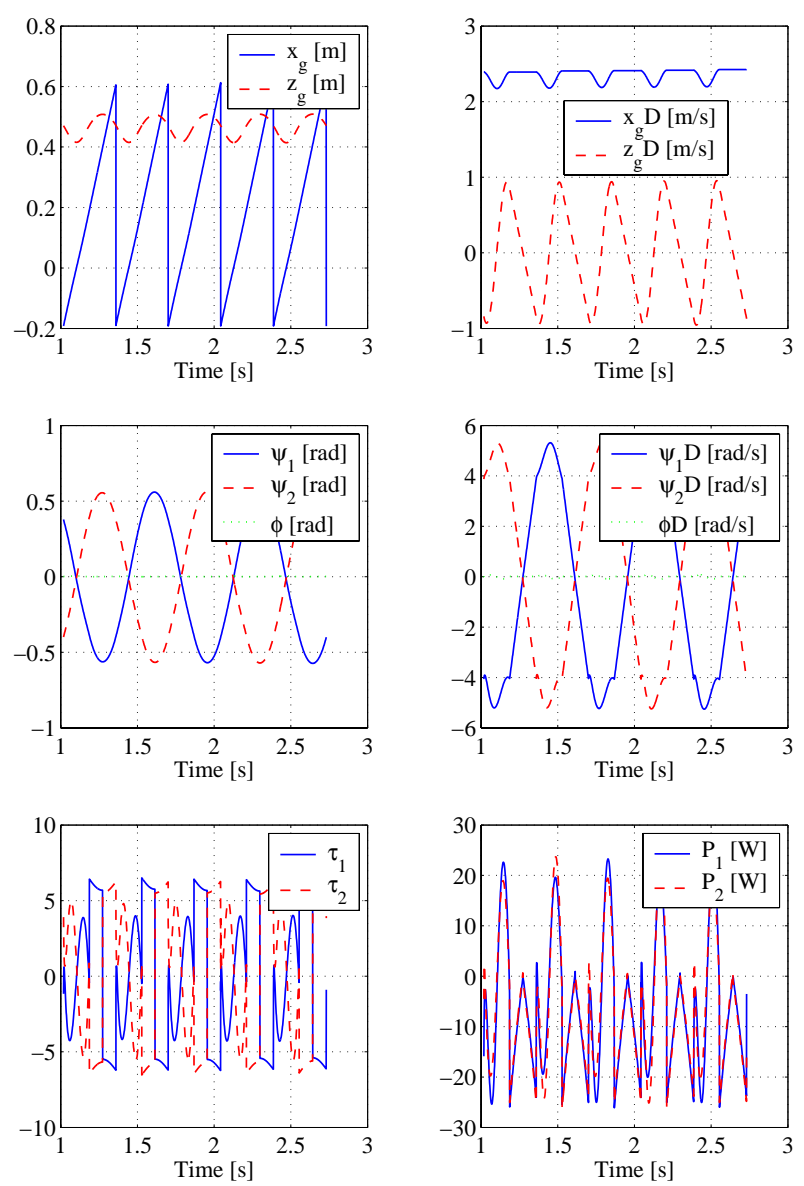

Fig. 6. Time evolutions of stable 1-periodic running gait. The top four graphs shows time evolutions of each state variable, where notation " $D$ " represents the time derivatives of preceding variables. $x_{g}$ is manually reset at each iterative crossing of the section, for visibility. The left bottom graph is the hip torques, while the right bottom graph shows power consumption at each hip actuator.
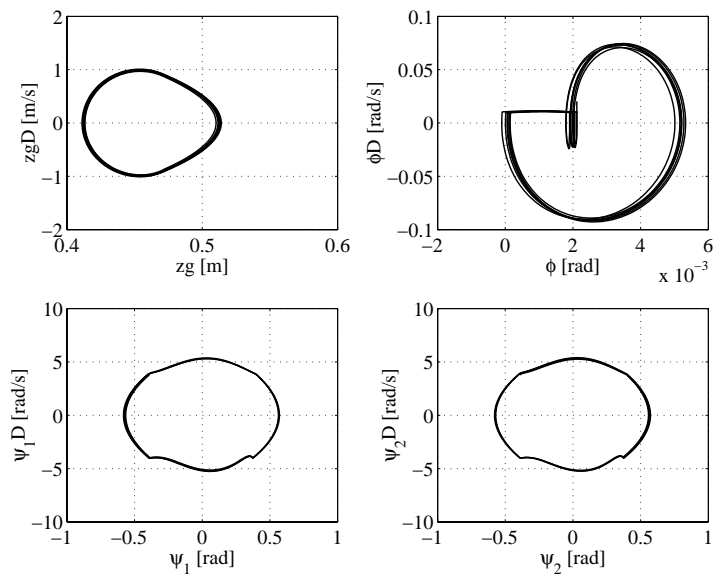

Fig. 7. Phase portraits of stable 1-periodic running gait. Notation “ $D$ " represents the time derivatives of preceding variables. Note that the scale of the abscissa of right top is $10^{-3}$ and small horizontal curve running form $\phi=0$ [rad] to $2 \times 10^{-3}$ [rad] does not mean an instantaneous jump, but indicates the result of high-gain PD-feedback about body pitch. 


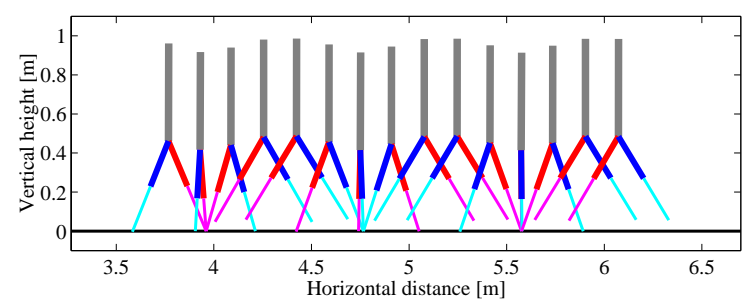

Fig. 8. Stic animation of subsequent three steps of 1-periodic biped running, corresponding Fig. 6. The robot moves from left to the right.
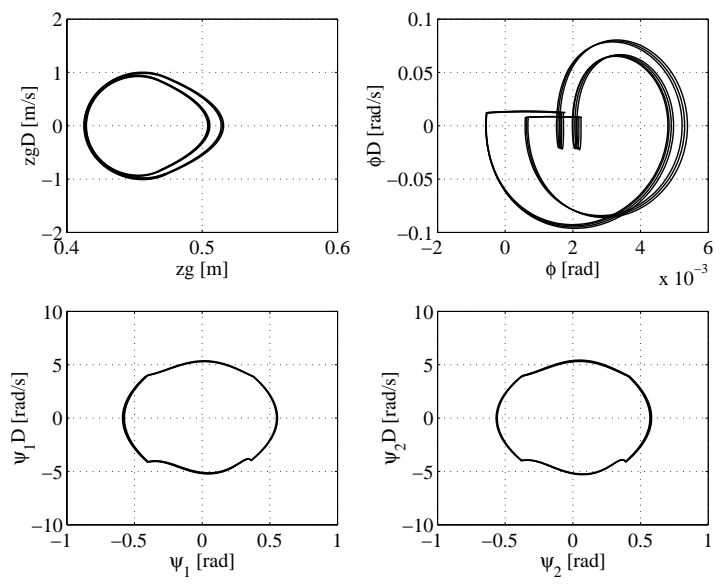

Fig. 9. Phase portraits of stable 2-periodic running gait. Notation " $D$ ” represents the time derivatives of preceding variables.

phase. In this simulation, the power consumption at hip joints exceeded 100 [W].

\section{CONCLUSION}

In this paper, we extended running controller of passive one-legged hopper to a planar biped robot with torso, and evaluated the controller on simulations. The controller was derived based on energy-preserving strategy and it actually preserved mechanical energy at touchdown phase, as in the case of one-legged model. Interestingly, zero dynamics of decoupling controller (dynamics about pair of controlled leg) was found to be stable. Combining simple attitude controller at stance phase generated stable periodic running gaits of arbitral period. The control performance was better than a simple PDfeedback control about leg placement.

The next task is to realize complete passive running gaits. To do so, we should introduce hip springs to make legs swung passively both at flight phase and stance phase. This implies replacing stance phase controller shown in Section III$\mathrm{B}$ with another stabilizing controller, where the control input eventually converges to zero. If this goal is achieved, the phase portraits of complete passive biped running gaits will exhibit strong symmetricity, as explained in [16]

Extending planar controller to 3D biped model is also important work. This was partially done by introducing one DOF at yaw-axis (rotor) in [17] and we are now adding more
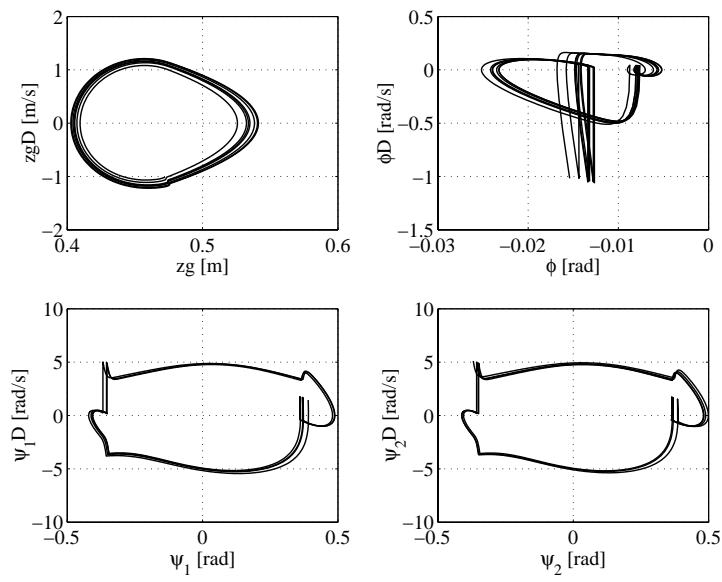

Fig. 10. Phase portraits of stable running gait using simple PD-feedback leg placement. Notation " $D$ " represents the time derivatives of preceding variables. In the bottom two figures, small vertical lines running form $\psi_{1,2}=$ $0[\mathrm{rad} / \mathrm{s}]$ to $5[\mathrm{rad} / \mathrm{s}]$ implies instantaneous velocity changes associated with touchdown impulse equation. This shows simple PD-feedback controller does not preserve energy at touchdown.

DOF at roll axis and applying the same controller. Although our controller cannot be applied to humanoid robot directly, we believe there is some embedding transformation.

\section{REFERENCES}

[1] M. Raibert: Legged Robots That Balance, MIT Press, 1985.

[2] R. Playter and M. Raibert: "Control of a biped somersault in 3D", Proc. of IEEE/RSJ IROS, pp.582-589, 1992.

[3] G. Zeglin and B. Brown: "Control of a bow leg hopping robot", Proc. of IEEE ICRA, pp.793-798, 1998.

[4] U. Saranli, M. Buehler and D.E. Koditschek: "RHex: a simple and highly mobile hexapod robot," Int. J. Robotics Research, vol.20, no.7, pp.616631, 2001.

[5] Y. Fukuoka, H. Kimura, et al.: "Adaptive dynamic walking of a quadruped robot 'Tekken' on irregular terrain using a neural system model', Proc. of IEEE ICRA, pp.2037-2042, 2003.

[6] S. Hyon and T. Mita: 'Development of a biologically inspired hopping robot -“Kenken"”, Proc. of IEEE ICRA, pp.3984-3991, 2002.

[7] R. M'Closkey and J. Burdick: "Periodic motions of a hopping robot with vertical and forward motion", Int. J. Robotics Research, vol.12, no.3, pp.197-218, 1993.

[8] W. Schwind and D. Koditschek: "Control of forward velocity for a simplified planar hopping robot", Proc. of IEEE ICRA, pp.691-696, 1995.

[9] C. Thompson and M. Raibert: "Passive dynamic running", Experimental Robotics I, eds. V.Hayward and O. Khatib., pp.74-83, Springer, 1989.

[10] M. Ahmadi and M. Buehler: "The ARL Monopod II running robot: control and energetics", Proc. of IEEE ICRA, pp.1689-1694, 1999.

[11] C. François and C. Samson: "A new approach to the control of the planar one-legged hopper", Int. J. Robotics Research, vol.17, no.11, pp.11501166, 1998.

[12] S.Hyon, T.Emura: "Quasi-periodic gaits of passive one-legged hopper", Proc. of IEEE/RSJ IROS, pp.2625-2630, 2002.

[13] Arnold, Mathematical Methods of Classical Mechanics, Springer, 1978.

[14] S. Hyon and T. Emura: 'Energy-preserving control of passive one-legged running robot", Advanced Robotics, vol.18, no.4, 2004 (to be published).

[15] K. Pyragas, "Continuous control of chaos by self-controlling feedback", Physi. Lett., A 170, pp.421-428, 1992.

[16] R. Altendorfer, D. E. Koditschek and P. Holmes: "Toward factored analysis of legged locomotion models", Proc. of IEEE ICRA, pp.37-44, 2003.

[17] S. Hyon and T. Emura: "Aerial posture control for 3D biped running using compensator around yaw axis", Proc. of IEEE ICRA, pp.57-62, 2003. 